\title{
Impact of the interval between coronary angiography and off-pump coronary bypass surgery on postoperative renal function
}

\author{
Na-young Kim, So Yeon Kim, Na Hyung Lee, and Young Lan Kwak* \\ Department of Anesthesiology and Pain Medicine, *Anesthesia and Pain Research Institute, Yonsei University College of Medicine, \\ Seoul, Korea
}

Background: Postoperative acute kidney injury (AKI) is a significant complication after coronary artery bypass surgery. Prior coronary angiography increases the likelihood of AKI due to the use of a radiocontrast dye. This study examined the effect of coronary angiography on the postoperative renal function after off-pump coronary artery bypass surgery (OPCAB).

Methods: The records of 110 patients who required OPCAB were reviewed. These patients also had at least two of the following conditions: chronic kidney disease, hypertension, diabetes mellitus, emergency surgery, congestive heart failure, age $>75$ years, hematocrit $<30 \%$, a left ventricular ejection fraction $<40 \%$, or the use of angiotensinconverting enzyme inhibitors or angiotensin receptor blockers. The patients were divided into two groups; coronary angiography performed within two days of OPCAB (Control group, $n=55$ ), and coronary angiography performed more than two days before ОРСАВ (Angio group, $\mathrm{n}=55$ ). The serum creatinine ( $\mathrm{SCr}$ ) and serum cystatin $\mathrm{C}$ levels were measured on the day before surgery, as well as on postoperative days $1,2,3$ and 7 . The estimated glomerular filtration rate (eGFR) was also obtained on those days. AKI was defined as an increase in $\mathrm{Cr} \geq 50 \%$ or $\geq 0.3 \mathrm{mg} / \mathrm{dl}$ within 48 hours.

Results: The postoperative changes in the SCr, cystatin C and eGFR were similar in the two groups. The incidence of AKI and renal replacement therapy were similar in the two groups.

Conclusions: Coronary angiography performed within two days of OPCAB does not affect the postoperative renal function. (Korean J Anesthesiol 2010; 58: 142-147)

Key Words: Coronary angiography, Kidney, Off-pump coronary artery bypass, Postoperative periods.

Received: October 16, 2009. Revised: 1st, October 27, 2009; 2nd, October 30, 2009. Accepted: December 17, 2009.

Corresponding author: Young Lan Kwak, M.D., Ph.D., Department of Anesthesiology and Pain Medicine, Anesthesia and Pain Research Institute, Yonsei University College of Medicine, 134, Shinchon-dong, Seodaemun-gu, Seoul 120-752, Korea. Tel: 82-2-2228-8513, Fax: 82-2-3642951, E-mail: ylkwak@yuhs.ac

(c)This is an open-access article distributed under the terms of the Creative Commons Attribution Non-Commercial License (http:// creativecommons.org/licenses/by-nc/3.0/), which permits unrestricted non-commercial use, distribution, and reproduction in any medium, provided the original work is properly cited. 


\section{Introduction}

Postoperative acute kidney injury (AKI), which was previously termed acute renal failure (ARF), is a serious and common complication after a coronary artery bypass graft (CABG). It is also associated with increased mortality [1-3], and the use of cardiopulmonary bypass $(\mathrm{CPB})$ is the greatest risk factor of AKI after CABG [4]. Before CABG, cardiac patients undergo coronary angiography using a radiocontrast dye, which can result in contrast-induced nephropathy (CIN). Accordingly, patients who had their coronary angiography performed in close proximity to surgery were at greater risk of developing postoperative AKI, suggesting the need to delay surgery after coronary angiography $[5]$.

By avoiding $\mathrm{CPB}$, patients undergoing off-pump CABG (OPCAB) can have a different level of risk of developing postoperative AKI despite their recent exposure to radiocontrast dye. However, there is no comprehensive data on the impact of the interval between coronary angiography and subsequent $\mathrm{OPCAB}$ on the postoperative renal function. It is important to determine if $\mathrm{OPCAB}$ is a safe alternative surgical option for patients with recent exposure to radiocontrast dye in terms of the development of postoperative AKI in order to avoid an unnecessary delay of CABG. This validation would be more evident if patients with the known risk factors for CIN, such as chronic kidney disease, hypertension, diabetes mellitus, emergency surgery, congestive heart failure, age $>75$ years, low hematocrit, left ventricular ejection fraction $<40 \%$, and use of angiotensin-converting enzyme inhibitors, are studied [6-8].

Therefore, this study evaluated retrospectively the impact of the interval between preoperative coronary angiography and OPCAB on the postoperative renal function in patients, who had more than two of these risk factors for CIN.

\section{Materials and Methods}

After gaining Institutional Review Board approval, the records from 110 patients who underwent OPCAB from March 1, 2008 to July 31, 2009 at our university hospital were reviewed. The demographics, clinical characteristics, comorbid conditions, medical treatments, laboratory data, angiographic data (including the amount and type of contrast used), cardiac procedural data (including its timing in relation to the preceding angiography), and peri- and postoperative events for all patients undergoing OPCAB were placed into a computerized database. The selected patients had coronary angiography followed by OPCAB at our institution and had at least two of the CIN risk factors (chronic kidney disease, hypertension, diabetes mellitus, emergency surgery, congestive heart failure, age $>75$ years, hematocrit $<30 \%$, left ventricular ejection fraction $<40 \%$, use of angiotensin-converting enzyme inhibitors or angiotensin receptor blockers). These inclusion criteria were used because the incidence of nephropathy requiring dialysis after percutaneous coronary intervention is higher in patients with more than two CIN risk factors [9]. In order to focus more on the impact of exposure to a radiocontrast on the postoperative renal function, patients with significant preoperative renal dysfunction (defined as serum $\mathrm{Cr}>1.8 \mathrm{mg}$ / dl) [10] or who received renal replacement therapy before surgery were excluded. Since CIN develops within 48 hours after administering the contrast media [11], two days was used as the dividing point for comparison. The patients were divided into the following two groups: coronary angiography performed within two days of OPCAB (Angio group, $n=55$ ) and coronary angiography performed more than two days before OPCAB (Control group, $\mathrm{n}=55$ ). The timing of surgery was dictated by the availability of an operating room slot, convenience and willingness of the patient to undergo surgery on a given day, rather than by a randomized assignment to a particular day; patient clinical condition after angiography; or the findings from angiography.

All patients received $0.05-0.1 \mathrm{mg} / \mathrm{kg}$ morphine intramuscularly as premedication $1 \mathrm{~h}$ before surgery. Upon arrival at the operating room, standard monitoring devices were applied. A pulmonary artery catheter (PAC, Swan-Ganz CCOmbo CCO/ $\mathrm{SvO}_{2}{ }^{\mathrm{TM}}$, Edwards Lifesciences LLC, Irvine, CA, USA), was inserted into the right internal jugular vein and connected to an analysis system (Vigilance ${ }^{\mathrm{TM}}$, Edwards Lifesciences LLC, Irvine, CA, USA) for continuous monitoring of the cardiac index (CI) and mixed venous oxygen saturation $\left(\mathrm{SvO}_{2}\right)$. Anesthesia was induced with intravenous midazolam (0.03-0.07 mg/ $\mathrm{kg}$ ) and sufentanil $(1.5-2.0 \mu \mathrm{g} / \mathrm{kg})$ and was maintained with sevoflurane $(0.8-1.5 \%)$ and a continuous infusion of sufentanil $(0.5-1.5 \mu \mathrm{g} / \mathrm{kg} / \mathrm{h})$. Neuromuscular blockade was achieved by administering rocuronium $(0.9 \mathrm{mg} / \mathrm{kg})$ and was maintained with a continuous infusion of vecuronium $(1-2 \mu \mathrm{g} / \mathrm{kg} / \mathrm{min})$. Isosorbide dinitrate $(0.5 \mu \mathrm{g} / \mathrm{kg} / \mathrm{min})$ was infused in all patients throughout the study period. After inducing anesthesia, a transesophageal echocardiography probe was inserted to assess the global cardiac function and detect newly developing segmental wall motion abnormalities. Intravascular volume replacement was managed with crystalloid and colloid solutions to maintain the pulmonary capillary wedge pressure in the range of 8-16 mmHg. During the period of heart displacement, the crystalloid solution was infused at a fixed rate of 6-8 $\mathrm{ml} /$ $\mathrm{kg}$, whereas the colloid solution was infused to compensate for the amount of blood loss, which was collected in a cell salvage device. The salvaged blood was reinfused into the patient before the completion of surgery. Hemodynamic management during the period of heart displacement and grafting was as follows: 1) 
maintenance of the mean systemic arterial pressure above 70 mmHg either with $10-15^{\circ}$ Trendelenburg positioning and/or norepinephrine infusion, 2) infusion of milrinone into patients with a $\mathrm{SvO}_{2}<60 \%$ for more than $10 \mathrm{~min}$ and/or the development of mitral regurgitation $\geq$ grade 3 with a concomitant $>30 \mathrm{mmHg}$ increase in mean pulmonary arterial pressure. Allogenic packed red blood cells were transfused when the hematocrit level was $<25 \%$ at any point within the study period. The central temperature, which was measured by a pulmonary artery catheter, was maintained above $36^{\circ} \mathrm{C}$ using a warm mattress, a forced warm air blanket and a fluid warmer where necessary.

All surgical procedures were performed by one surgeon through a median sternotomy, and the heart was displaced using a posterior pericardial stitch, large $(12 \times 70 \mathrm{~cm})$ gauze swabs and a tissue stabilizer (Rosta ${ }^{\circledR} 2.0$, Yoorim Corporation, Chungbuck, Korea). The sequence of grafting was always from the left internal mammary artery to the left anterior descending coronary artery (LAD) first, followed by grafting of the circumflex coronary artery (LCx) and right coronary artery (RCA) using a composite Y graft consisting of the radial artery or saphenous vein with the left internal mammary artery and/ or using the right internal mammary artery where necessary. An intracoronary shunt was used on the LAD and distal RCA during grafting. All patients were transferred to the intensive care unit (ICU) after surgery.

The preoperative data included age, gender, body mass index, as well as the presence of diabetes mellitus, hypertension, or chronic renal disease. The other preoperative data included cardiac medications, electrocardiography, echocardiographic data (left ventricular ejection fraction and E/e'), and volume of the radiocontrast solution. The intraoperative data obtained included the duration of anaesthesia and the number of grafts performed. In the ICU, the following variables were recorded: postoperative day one blood loss, duration of ventilation, length of ICU stay and length of hospital stay. Also included were the occurrence of re-intubation, the need for hemostatic reexploration, and the occurrence of postoperative atrial fibrillation and/or death.

The serum creatinine (SCr), serum cystatin $\mathrm{C}$ and estimated glomerular filtration rate (eGFR) were obtained one day before surgery and on postoperative days (POD) 1, 2, 3 and 7 . This information was used to evaluate the renal function. The postoperative AKI was diagnosed if there was $\geq 50 \%$ or $\geq 0.3$ $\mathrm{mg} / \mathrm{dl}$ increase in the serum creatinine level within 48 hours, compared to the preoperative baseline value [12].

Statistical analysis was performed using SPSS 13.0 (SPSS Inc., Chicago, IL, USA). Forty-five patients were required in each group to produce an $80 \%$ power to detect a $0.3 \mathrm{mg} / \mathrm{dl}$ difference in Cr between the two groups with a SD of $0.5 \mathrm{mg} /$ $\mathrm{dl}$ and an alpha level of $0.05 \mathrm{in}$ an independent t-test [13]. All the data is expressed as the mean $\pm \mathrm{SD}$, median (interquartile range) or number of patients (\%) according to the distribution of normality. The data between the groups was compared using a Chi-square test, Fisher's exact test, independent t-test or a Mann-Whitney U-test, where appropriate. Repeated measures analysis of variance was performed to compare the changes in SCr, cystatin $\mathrm{C}$ and eGFR between the groups. A P value $<0.05$ was considered statistically significant.

\section{Results}

In all patients, iopromide (Ultravist ${ }^{\circledR}$; Bayer Schering, Seoul, Korea) or iodixanol (Visipaque ${ }^{\circledR}$; GE Healthcare, Seoul, Korea) was used as a contrast agent during coronary angiography. The patients' characteristics including the number of CIN risk factors were similar in the two groups (Table 1). In the Angio

Table 1. Patient Characteristics

\begin{tabular}{|c|c|c|c|}
\hline & $\begin{array}{l}\text { Control } \\
(n=55)\end{array}$ & $\begin{array}{l}\text { Angio } \\
(\mathrm{n}=55)\end{array}$ & $P$ value \\
\hline Age (yr) & $65 \pm 9$ & $67 \pm 8$ & 0.163 \\
\hline $\operatorname{Sex}(M / F)$ & $41: 14$ & $41: 14$ & 1.000 \\
\hline Body mass index $\left(\mathrm{m}^{2}\right)$ & $24.5 \pm 3.0$ & $24.9 \pm 2.9$ & 0.451 \\
\hline NYHA grade I-II & $48(87)$ & $44(80)$ & \\
\hline grade III-IV & $7(13)$ & $11(20)$ & 0.303 \\
\hline Hypertension & $41(75)$ & $48(87)$ & 0.089 \\
\hline Diabetes mellitus & $32(58)$ & $27(49)$ & 0.339 \\
\hline Chronic renal disease & $1(2)$ & $2(4)$ & 1.000 \\
\hline Preoperative hematocrit & $37.2 \pm 5.4$ & $37.9 \pm 4.9$ & 0.491 \\
\hline Elective: emergency surgery & $53: 2$ & $50: 5$ & 0.438 \\
\hline Number of CIN risk factors & $3(2-3)$ & $2(2-4)$ & 0.786 \\
\hline $\begin{array}{l}\text { Electrocardiography } \\
\text { (NSR : A-fib) }\end{array}$ & $55: 0$ & $54: 1$ & 1.000 \\
\hline LVEF (percent) & $55(36-67)$ & $59(45-68)$ & 0.247 \\
\hline $\mathrm{E} / \mathrm{e}^{\prime}$ & $13(10-17)$ & $13(10-17)$ & 0.592 \\
\hline \multicolumn{4}{|l|}{ Medications } \\
\hline ACEI & $22(40)$ & $17(31)$ & 0.319 \\
\hline ARB & $23(42)$ & $26(47)$ & 0.565 \\
\hline Diuretics & $11(20)$ & $5(9)$ & 0.105 \\
\hline Number of grafts & $3(3-4)$ & $3(3-4)$ & 0.881 \\
\hline Duration of anesthesia (min) & $302 \pm 40$ & $307 \pm 49$ & 0.547 \\
\hline Volume of contrast (ml) & $123(100-144)$ & $123(100-150)$ & 0.467 \\
\hline
\end{tabular}

Values are reported as the mean $\pm \mathrm{SD}$, median (interquartile range) or number of patients (\%). Control: coronary angiography performed within two days of OPCAB, Angio: coronary angiography performed more than two days before OPCAB, NYHA: New York Heart Association, LVEF: left ventricular ejection fraction, CIN: contrast-induced nephropathy, NSR: normal sinus rhythm, A-fib: atrial fibrillation, ACEI: angiotensin-converting enzyme inhibitor, ARB: angiotension receptor blocker. 
group, 5, 17 and 33 patients received coronary angiography on the same day of surgery, one day and two days before surgery, respectively. In the Control group, coronary angiography was performed three to 69 days before OPCAB.

Intraoperative fluid management, transfusion or the use of vasopressors that can affect postoperative renal function were similar in the two groups (Table 2). The preoperative SCr, cystatin $\mathrm{C}$ and eGFR were similar in the two groups throughout the study period (Table 3). Fig. 1 shows the incidence of postoperative AKI and renal replacement therapy. Eight (15\%) patients in the Control group and ten (18\%) patients in the Angio group developed AKI. One patient in the Control group and four patients in the Angio group required renal replacement therapy postoperatively. Although the Angio group had a higher incidence of AKI and renal replacement therapy, it was not statistically significant $(\mathrm{P}=0.667$ and 0.363 , respectively).

The median duration of the ICU stay was significantly longer in the Angio group than the Control group [52.0 (IQR 48.0-75.0) vs. 48.9 (IQR 44.8-71.3) h, $\mathrm{P}=0.048$ ], even though the duration of the hospital stay was similar (Table 4). There were no significant differences in the other postoperative outcomes.

Table 2. Intraoperative Administration of Fluid and Red Blood Cell and the Use of Vasopressor

\begin{tabular}{lccc}
\hline & Control $(\mathrm{n}=55)$ & Angio $(\mathrm{n}=55)$ & P value \\
\hline Crystalloid amount $(\mathrm{ml})$ & 2,300 & 2,500 & 0.104 \\
& $(1,800-2,600)$ & $(2,200-2,900)$ & \\
Colloid amount (ml) & 1,300 & 1,300 & 0.347 \\
& $(1,200-1,500)$ & $(1,100-1,500)$ & \\
Total fluid amount (ml) & 3,650 & 3,800 & 0.225 \\
& $(3,100-4,100)$ & $(3,200-4,300)$ & \\
RBC transfusion & $21(38 \%)$ & $19(35 \%)$ & 0.632 \\
Urine output (ml) & $520(345-760)$ & $660(420-900)$ & 0.102 \\
Use of vasopressor & $53(96 \%)$ & $53(96 \%)$ & 1.000 \\
\hline
\end{tabular}

Values are reported as the median (interquartile range) or number of patients (\%). Control: coronary angiography performed within two days of OPCAB, Angio: coronary angiography performed more than two days before OPCAB.

\section{Discussion}

This study examined the effect of the time interval between coronary angiography and OPCAB on the postoperative renal function. Coronary angiography performed within two days before OPCAB did not adversely affect the postoperative renal function in terms of the postoperative SCr, cystatin $\mathrm{C}$ and eGFR levels or the incidence of postoperative AKI. Since CIN develops within 48 hours after angiography [11], the incidence of postoperative renal dysfunction was expected to be higher in those patients who had undergone angiography within two days of OPCAB. These findings are in contrast to previous studies with patients undergoing CABG using CPB [5,14]. Although cardiac surgery is generally performed or postponed for days or weeks after coronary angiography because of the concerns about the development of CIN, the results of this study suggest that choosing to delay OPCAB due to the limited passage of time since coronary angiography might not be necessary in patients with a preoperatively normal renal function.

A renal dysfunction is one of the most common and serious

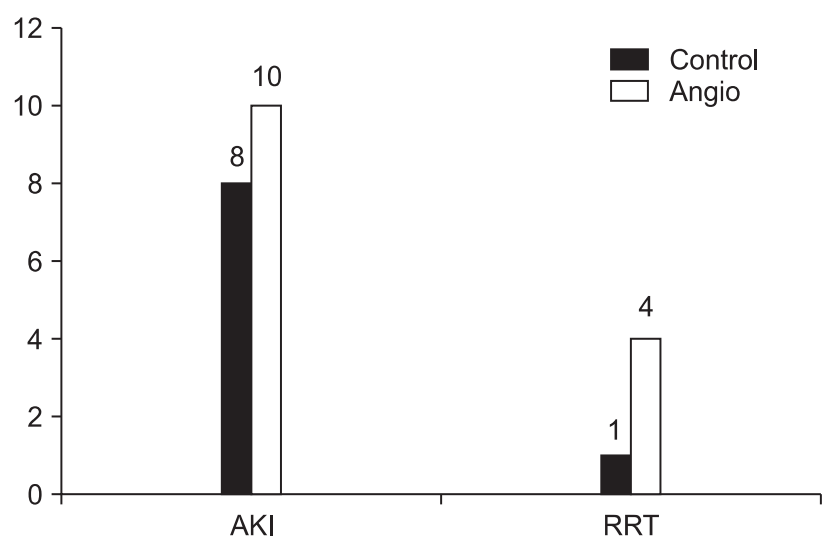

Fig. 1. Incidence of acute kidney injury and renal replacement therapy. Control: coronary angiography performed within two days of $\mathrm{OPCAB}$, Angio: coronary angiography performed more than two days before OPCAB, AKI: acute kidney injury, RRT: renal replacement therapy.

Table 3. Changes in the Serum Creatinine, Cystatin C, and Estimated GFR Levels during the Perioperative Period

\begin{tabular}{|c|c|c|c|c|c|c|}
\hline & Group & Pre-operation & POD 1 & POD 2 & POD 3 & POD 7 \\
\hline \multirow[t]{2}{*}{ Serum Cr (mg/dl) } & Control & $1.00(0.85-1.20)$ & $1.00(0.80-1.20)$ & $1.04(0.90-1.27)$ & $1.08(0.93-1.33)$ & $1.00(0.86-1.18)$ \\
\hline & Angio & $0.99(0.88-1.20)$ & $0.95(0.79-1.21)$ & $1.03(0.83-1.39)$ & $1.11(0.86-1.11)$ & $1.04(0.90-1.31)$ \\
\hline \multirow[t]{2}{*}{ Serum cystatin C (mg/L) } & Control & $0.83(0.74-0.97)$ & $0.67(0.57-0.81)$ & $0.78(0.66-1.00)$ & $0.85(0.70-0.99)$ & $0.85(0.75-1.03)$ \\
\hline & Angio & $0.85(0.67-1.03)$ & $0.77(0.56-0.94)$ & $0.84(0.62-1.17)$ & $0.94(0.74-1.15)$ & $1.06(0.79-1.30)$ \\
\hline \multirow[t]{2}{*}{$\mathrm{eGFR}\left(\mathrm{ml} / \mathrm{min} / 1.73 \mathrm{~m}^{2}\right)$} & Control & $74.5(61.0-87.1)$ & $73.3(59.3-98.8)$ & $70.3(57.1-82.2)$ & $71.5(52.9-83.2)$ & $76.8(62.2-88.8)$ \\
\hline & Angio & $74.6(57.9-87.9)$ & $77.1(58.8-98.9)$ & $68.9(51.1-91.2)$ & $69.1(47.9-89.7)$ & $69.5(51.5-86.9)$ \\
\hline
\end{tabular}

Values are reported as the median (interquartile range). Control: coronary angiography performed within two days of OPCAB, Angio: coronary angiography performed more than two days before OPCAB, Cr: creatinine, eGFR: estimated glomerular filtration rate, POD: postoperative day. 
Table 4. Postoperative Outcomes

\begin{tabular}{|c|c|c|c|}
\hline & Control $(n=55)$ & Angio $(\mathrm{n}=55)$ & $\mathrm{P}$ value \\
\hline $\begin{array}{l}\text { Duration of ventilation in } \\
\text { ICU (h) }\end{array}$ & $\begin{array}{c}17.2 \\
(15.4-18.0)\end{array}$ & $\begin{array}{c}17.7 \\
(14.5-19.0)\end{array}$ & 0.704 \\
\hline Duration of ICU stay (h) & $\begin{array}{c}48.9 \\
(44.8-71.3)\end{array}$ & $\begin{array}{c}52.0 \\
(48.0-75.0)^{*}\end{array}$ & 0.048 \\
\hline $\begin{array}{l}\text { Duration of hospital stay } \\
\text { after operation (d) }\end{array}$ & $\begin{array}{c}9.0 \\
(9.0-12.5)\end{array}$ & $\begin{array}{c}9.0 \\
(7.0-10.0)\end{array}$ & 0.122 \\
\hline Re-intubation & $1(2)$ & $1(2)$ & 1.000 \\
\hline Hemostatic re-exploration & 0 & $1(2)$ & 1.000 \\
\hline Death & $1(2)$ & $2(4)$ & 1.000 \\
\hline $\begin{array}{l}\text { Postoperative day } 1 \text { blood } \\
\text { loss }\end{array}$ & $980(720-1,330)$ & $810(530-1,025)$ & 0.098 \\
\hline Postoperative A-fib & $3(6)$ & $8(15)$ & 0.122 \\
\hline
\end{tabular}

Values are reported as the mean $\pm \mathrm{SD}$, median (interquartile range) or number of patients (\%). Control: coronary angiography performed within two days of $\mathrm{OPCAB}$, Angio: coronary angiography performed more than two days before OPCAB, A-fib: atrial fibrillation. ${ }^{*} \mathrm{P}<0.05$ versus control group.

complications after CABG [1-4]. Even small increases in SCr are associated with increased mortality [1-3]. The most important factors contributing to postoperative renal dysfunction after cardiac surgery are the use of $\mathrm{CPB}$ and other factors associated with using CPB [4]. Therefore, despite the controversy, OPCAB performed without $\mathrm{CPB}$ is expected to have a positive effect on the postoperative renal function. The incidence of AKI was lower in $\mathrm{OPCAB}$ patients than in those who had undergone CABG using CPB (14\% vs. 24\%) [15]. These findings are comparable to the results of this study, but higher than the $7.5 \%$ of non-cardiac surgery [16]. Recently, a definition of AKI was established by the consensus of all key critical care and nephrology societies worldwide [12], and the new definition of AKI was applied in this study. The possible reason for the renal dysfunction after OPCAB was attributed to the impaired renal perfusion during distal anastomosis of the lateral and posterior vessels requiring verticalization of the heart and a resulting outflow tract obstruction and low cardiac output [17].

One of the new concerns in renal dysfunction after cardiac surgery is the timing of coronary angiography, because coronary angiography can affect the renal function. The SCr level begins to increase within $24 \mathrm{~h}$ of contrast administration and usually peaks within 3-5 days [18]. Therefore, the timing of coronary angiography can affect the postoperative SCr levels. Few studies have evaluated the impact of coronary angiography on the postoperative renal function in cardiac surgery using CPB $[5,10,14]$. In two of these studies, the same day surgery and coronary angiography [14], and coronary angiography performed within 5 days before surgery [5] increased the peak postoperative SCr levels and the risk of ARF. However, in a report from the Mayo Clinic [10], same-day coronary angiography had little impact on the renal function for patients undergoing elective valvular heart surgery. The much shorter duration of CPB (48 $\pm 20 \mathrm{~min}$ ) compared to the former studies might have little impact on the renal function in that study. Postoperative renal dysfunction after cardiac surgery was closely related to the duration of CPB [5]. The subsequent exposure to CPB after the use of a contrast agent can have a synergistically adverse effect on the postoperative renal function. Therefore, the impact of the timing of angiography might be different between cardiac surgery using $\mathrm{CPB}$ and $\mathrm{OPCAB}$. The differences in patient selection can also contribute to the differences in the results. In this study, patients with a preoperative SCr level exceeding 1.8 $\mathrm{mg} / \mathrm{dl}$ were excluded, which is unlike previous studies which excluded only those patients on dialysis before surgery $[5,14]$. On the other hand, in the present study, patients with at least two risk factors for CIN were included in order to focus more on the impact of exposure to the radiocontrast on the postoperative renal function.

Although SCr was used as a guide for a diagnosis of AKI, eGFR was also evaluated as a renal function index, as in previous studies $[5,14]$. The cystatin C levels were also monitored. The SCr levels are strongly influenced by the muscle mass, and eGFR is not optimal in people with normal SCr levels [19]. However, eGFR was recommended as an appropriate renal function measure by the National Kidney Foundation [20]. Cystatin C is believed to overcome some of the limitations of SCr and eGFR, as it does not depend on age, gender or body mass. However, AKI has not been defined using the cystatin C levels. In patients with $\mathrm{AKI}$, the cystatin $\mathrm{C}$ levels increased before SCr and allowed for the detection of AKI one to two days earlier than SCr [21]. In this study, there were no differences in the cystatin $C$ levels during the postoperative period between the two groups.

Contrast media are commonly divided into high-osmolar, low-osmolar and iso-osmolar. Iopromide is one of the lowosmolar contrast media. Meta-analysis suggests that lowosmolar contrast media caused a smaller decrease in renal function than the high-osmolar contrast media [22]. In addition, it was demonstrated that the iso-osmolar contrast media (iodixanol) exhibits lower nephrotoxic properties than the lowosmolar media in a population of patients at very high risk [23]. In this study, two types of radiocontrast, iopromide or iodixanol, were used in the patients. The use of less nephrotoxic contrast media might be a possible reason for little impact of the timing of angiography on AKI. The prophylaxis strategy for CIN (e.g., adequate hydration, minimal volume of iso-osmolar or lowosmolar contrast, $\mathrm{N}$-acetylcystein) can be helpful in reducing the incidence of AKI in patients with the risk factors for CIN [24]. Moreover, considering that the incidence of AKI was still high in this study, careful compression and positioning of the heart during distal anastomosis to minimize hemodynamic 
instability and maintain adequate renal perfusion [17] might be more important for reducing the incidence of AKI in patients undergoing OPCAB than the timing of angiography.

This study was limited by potentially confounding information and selection bias, which are the same pitfalls encountered in previous studies. However, previous studies $[5,10,14]$ did not mention important factors, such as intraoperative fluid management, transfusion or use of vasopressors, which can affect postoperative renal function. This study controlled the possible factors affecting renal functions according to institutional guidelines. The sample size was calculated according to the difference in $\mathrm{SCr}$, and might be too small to allow for the detection of differences in the incidence of AKI and renal replacement therapy (RRT). Despite the similar postoperative outcome variables, the patients in the Angio group stayed longer in the ICU than the Control group. Although the precise reason for this longer ICU stay could not be identified, it might be due to a trend of more frequent RRT in the Angio group because RRT is a well known contributing factor for a prolonged stay in the ICU. Indeed, the duration of the ICU stay was similar in the two the groups when the patients undergoing RRT were excluded ( $\mathrm{P}=0.092)$.

In conclusion, coronary angiography performed within two days before OPCAB did not affect the postoperative renal function and incidence of AKI in patients with a preoperatively normal renal function. These findings suggest that there is no need to delay OPCAB after using a radiocontrast agent due to the concern of AKI in patients with a preoperatively normal renal function.

\section{References}

1. Aronson S, Fontes ML, Miao Y, Mangano DT. Risk index for perioperative renal dysfunction/failure: critical dependence on pulse pressure hypertension. Circulation 2007; 115: 733-42.

2. Loef BG, Epema AH, Smilde TD, Henning RH, Ebels T, Navis G, et al. Immediate postoperative renal function deterioration in cardiac surgical patients predicts in-hospital mortality and long-term survival. J Am Soc Nephrol 2005; 16: 195-200.

3. Ryckwaert F, Boccara G, Frappier JM, Colson PH. Incidence, risk factors, and prognosis of a moderate increase in plasma creatinine early after cardiac surgery. Crit Care Med 2002; 30: 1495-8.

4. Rosner MH, Okusa MD. Acute kidney injury associated with cardiac surgery. Clin J Am Soc Nephrol 2006; 1: 19-32.

5. Del Duca D, Iqbal S, Rahme E, Goldberg P, de Varennes B. Renal failure after cardiac surgery: timing of cardiac catheterization and other perioperative risk factors. Ann Thorac Surg 2007; 84: 1264-71.

6. Bartholomew BA, Harjai KJ, Dukkipati S, Boura JA, Yerkey MW, Glazier S, et al. Impact of nephropathy after percutaneous coronary intervention and a method for risk stratification. Am J Cardiol 2004; 93: 1515-9.

7. Mehran R, Aymong ED, Nikolsky E, Lasic Z, Iakovou I, Fahy M, et al.
A simple risk score for prediction of contrast-induced nephropathy after percutaneous coronary intervention: development and initial validation. J Am Coll Cardiol 2004; 44: 1393-9.

8. Nikolsky E, Aymong ED, Dangas G, Mehran R. Radiocontrast nephropathy: identifying the high-risk patient and the implications of exacerbating renal function. Rev Cardiovasc Med 2003; 4 Suppl 1: S7-14.

9. Freeman RV, O'Donnell M, Share D, Meengs WL, Kline-Rogers E, Clark VL, et al. Nephropathy requiring dialysis after percutaneous coronary intervention and the critical role of an adjusted contrast dose. Am J Cardiol 2002; 90: 1068-73.

10. Brown ML, Holmes DR, Tajik AJ, Sarano ME, Schaff HV. Safety of same-day coronary angiography in patients undergoing elective valvular heart surgery. Mayo Clin Proc 2007; 82: 572-4.

11. Toprak $\mathrm{O}$. What is the best definition of contrast-induced nephropathy? Ren Fail 2007; 29: 387-8.

12. Mehta RL, Kellum JA, Shah SV, Molitoris BA, Ronco C, Warnock DG, et al. Acute Kidney Injury Network: report of an initiative to improve outcomes in acute kidney injury. Crit Care 2007; 11: R31.

13. Ogawa M, Yaku H, Doi K, Okano T, Yamada Y, Hayashida K, et al. Does off-pump coronary artery bypass grafting beneficially affect renal function? ANZ J Surg 2005; 75: 675-9.

14. Ranucci M, Ballotta A, Kunkl A, De Benedetti D, Kandil H, Conti D, et al. Influence of the timing of cardiac catheterization and the amount of contrast media on acute renal failure after cardiac surgery. Am J Cardiol 2008; 101: 1112-8.

15. Massoudy P, Wagner S, Thielmann M, Herold U, KottenbergAssenmacher E, Marggraf G, et al. Coronary artery bypass surgery and acute kidney injury--impact of the off-pump technique. Nephrol Dial Transplant 2008; 23: 2853-60.

16. Abelha FJ, Botelho M, Fernandes V, Barros H. Determinants of postoperative acute kidney injury. Crit Care 2009; 13: R79.

17. Patel NC, Grayson AD, Jackson M, Au J, Yonan N, Hasan R, et al. The effect off-pump coronary artery bypass surgery on in-hospital mortality and morbidity. Eur J Cardiothorac Surg 2002; 22: 255-60.

18. Gleeson TG, Bulugahapitiya S. Contrast-induced nephropathy. AJR Am J Roentgenol 2004; 183: 1673-89.

19. Verhave JC, Gansevoort RT, Hillege HL, De Zeeuw D, Curhan GC, De Jong PE. Drawbacks of the use of indirect estimates of renal function to evaluate the effect of risk factors on renal function. J Am Soc Nephrol 2004; 15: 1316-22.

20. K/DOQI clinical practice guidelines for chronic kidney disease: evaluation, classification, and stratification. Am J Kidney Dis 2002; 39: S1-266.

21. Herget-Rosenthal S, Marggraf G, Husing J, Goring F, Pietruck F, Janssen O, et al. Early detection of acute renal failure by serum cystatin C. Kidney Int 2004; 66: 1115-22.

22. Barrett BJ, Carlisle EJ. Metaanalysis of the relative nephrotoxicity of high- and low-osmolality iodinated contrast media. Radiology 1993; 188: 171-8.

23. Aspelin P, Aubry P, Fransson SG, Strasser R, Willenbrock R, Berg KJ. Nephrotoxic effects in high-risk patients undergoing angiography. $\mathrm{N}$ Engl J Med 2003; 348: 491-9.

24. Pannu N, Wiebe N, Tonelli M. Prophylaxis strategies for contrastinduced nephropathy. JAMA 2006; 295: 2765-79. 